MATHEMATICS OF COMPUTATION

Volume 71, Number 239, Pages 1243-1262

S 0025-5718(02)01395-9

Article electronically published on January 11, 2002

\title{
EVALUATION OF ZETA FUNCTION OF THE SIMPLEST CUBIC FIELD AT NEGATIVE ODD INTEGERS
}

\author{
HYUN KWANG KIM AND JUNG SOO KIM
}

\begin{abstract}
In this paper, we are interested in the evaluation of the zeta function of the simplest cubic field. We first introduce Siegel's formula for values of the zeta function of a totally real number field at negative odd integers. Next, we will develop a method of computing the sum of a divisor function for ideals, and will give a full description for a Siegel lattice of the simplest cubic field. Using these results, we will derive explicit expressions, which involve only rational integers, for values of a zeta function of the simplest cubic field. Finally, as an illustration of our method, we will give a table for zeta values for the first one hundred simplest cubic fields.
\end{abstract}

\section{INTRODUCTION}

Using finite dimensionality of elliptic modular forms of weight $h$, Siegel [7] developed an ingenious method of computing $\zeta_{K}(b)$, where $K$ is a totally real algebraic number field, $\zeta_{K}(s)$ is the Dedekind zeta function of $K$, and $b$ is a negative odd integer. However, evaluation of values of a zeta function by means of Siegel's formula requires complicated computations in algebraic number theory, since the formula involves terminology of algebraic number theory, such as norm, trace and different of $K$. The problem of expressing zeta values in terms of elementary functions was first studied by Zagier [10]. Siegel's formula has been exploited by Zagier to give an elementary expression for $\zeta_{K}(1-2 s)$, where $K$ is a real quadratic field and $s$ is a positive integer, which involves only rational integers and not algebraic numbers or norm of ideals. In this paper, we will be interested in expressing zeta values of a certain class of totally real cyclic cubic fields, which are called the simplest cubic fields, in terms of elementary functions.

It is well known (cf. [5, Appendix A.3]) that every cyclic cubic field can be obtained by adjoining to $\mathbb{Q}$ a root of an irreducible polynomial

$$
f(x)=x^{3}+m x^{2}-(m+3) x+1,
$$

where $m$ runs over the set of rational numbers. Let $K_{m}$ (or simply $K$ ) denote the cyclic cubic field corresponding to $m \in \mathbb{Q}$. Since $K_{m}$ and $K_{-m-3}$ represent the same field, we may assume that $m \geq-\frac{3}{2}$. The discriminant of the polynomial $f(x)$

Received by the editor July 25, 2000 and, in revised form, September 26, 2000.

2000 Mathematics Subject Classification. Primary 11R42; Secondary 11R16.

Key words and phrases. The simplest cubic field, zeta function, Siegel lattice.

The present studies were supported by the Korea Research Foundation Grant (KRF-97-001D00011-D1101) and Com² MaC-KOSEF. 
is $D^{2}$, where $D=m^{2}+3 m+9$. Let $\rho$ be the negative root of $f(x)$. Then

$$
\rho^{\prime}=\frac{1}{1-\rho}, \quad \rho^{\prime \prime}=1-\frac{1}{\rho}
$$

are the other roots of $f(x)$ so that $K=\mathbb{Q}(\rho)$ is a cyclic cubic field. The terminology "simplest cubic field" goes back to a work of Shanks [6]. He studied the arithmetic of a family of cyclic cubic fields which corresponds to $m \in \mathbb{Z}$ such that $D=m^{2}+3 m+9$ is a prime, and he called these fields the simplest cubic fields. The notion was extended by Washington 8 in which he studied the arithmetic of a family of cyclic cubic fields which corresponds to $m \in \mathbb{Z}, m \not \equiv 3(\bmod 9)$. The simplest cubic field in the sense of this paper means that it corresponds to $m \in \mathbb{Z}$ such that $D=m^{2}+3 m+9$ is square-free. In this case, we have

Proposition 1.1. Let $m(\geq-1)$ be an integer such that $D=m^{2}+3 m+9$ is squarefree. Then $\left\{1, \rho, \rho^{2}\right\}$ forms an integral basis of $K$ and $\left\{-1, \rho, \rho^{\prime}\right\}$ generates the full unit group of $K$.

Proof. See [8].

In this paper, we shall apply Siegel's formula to the simplest cubic field $K$ to obtain an elementary expression of $\zeta_{K}(1-2 s)$. In Section 2, we will introduce Siegel's formula and the notion of a Siegel lattice. In Section 3, we will express the sum of an ideal divisor function $\sigma_{r}(\mathfrak{A})$ in terms of the usual sum of divisor function $\sigma_{r}(n)$. In Section 4, we shall describe a Siegel lattice for the simplest cubic field. In Section 5, we will obtain a formula for the values of the zeta function of $K$ which involves only rational integers. Finally, as an illustration of our computation, we will compute $\zeta_{K}(-1), \zeta_{K}(-3)$, and $\zeta_{K}(-5)$ for the first one hundred values of corresponding $m$ 's.

\section{Siegel's formula And a Siegel lattice}

In this section, we first state Siegel's formula for values of the zeta function of a totally real algebraic number field at negative odd integers. Next, we discuss what is needed to apply Siegel's formula for the computation of values of the zeta function. Finally, we introduce the notion of a Siegel lattice which will be crucial in our computation.

Let $K$ be an algebraic number field and $\mathcal{O}_{K}$ be the ring of integers of $K$. For an ideal $\mathfrak{A}$ of $\mathcal{O}_{K}$, we define the sum of divisors function $\sigma_{r}(\mathfrak{A})$ by setting

$$
\sigma_{r}(\mathfrak{A})=\sum_{\mathfrak{B} \mid \mathfrak{A}} N_{K / \mathbb{Q}}(\mathfrak{B})^{r}
$$

where $\mathfrak{B}$ runs over all ideals of $\mathcal{O}_{K}$ which divide $\mathfrak{A}$. Note that, if $K=\mathbb{Q}$ and $\mathfrak{A}=(n)$, our definition coincides with the usual sum of the divisor function

$$
\sigma_{r}(n)=\sum_{\substack{d \mid n \\ d>0}} d^{r}
$$

Now let $K$ be a totally real algebraic number field. For $l, s=1,2, \ldots$, we define

$$
S_{l}^{K}(2 s)=\sum_{\substack{\nu \in \delta-1 \\ \nu \gg 0 \\ \operatorname{tr}(\nu)=l}} \sigma_{2 s-1}((\nu) \delta),
$$


where $\delta$ denotes the different of $K$. Later we shall study the sum (3) intensively. At this moment, we remark that this is a finite sum.

We now state Siegel's formula.

Theorem 2.1 (Siegel). Let $s=1,2, \ldots$, be a natural number, $K$ a totally real algebraic number field of degree $n$, and $h=2 s n$. Then

$$
\zeta_{K}(1-2 s)=2^{n} \sum_{l=1}^{r} b_{l}(h) S_{l}^{K}(2 s)
$$

The numbers $r \geq 1$ and $b_{1}(h), \ldots, b_{r}(h) \in \mathbb{Q}$ depend on $h$. In particular,

$$
r=\operatorname{dim}_{\mathbb{C}} \mathfrak{M}_{h},
$$

where $\mathfrak{M}_{h}$ denotes the space of modular forms of weight $h$. Thus by a well-known formula,

$$
r=\left\{\begin{array}{lll}
{\left[\frac{h}{12}\right]} & \text { if } h \equiv 2 \quad(\bmod 12) \\
{\left[\frac{h}{12}\right]+1} & \text { if } \quad h \neq 2 \quad(\bmod 12)
\end{array}\right.
$$

Proof. See [7] or [10].

Remark. By applying (4) to the simplest cubic field $K$, we obtain

$$
\begin{aligned}
& \zeta_{K}(-1)=2^{3} * b_{1}(6) * S_{1}^{K}(2), \\
& \zeta_{K}(-3)=2^{3} *\left[b_{1}(12) * S_{1}^{K}(4)+b_{2}(12) * S_{2}^{K}(4)\right], \\
& \zeta_{K}(-5)=2^{3} *\left[b_{1}(18) * S_{1}^{K}(6)+b_{2}(18) * S_{2}^{K}(6)\right] .
\end{aligned}
$$

Zagier [10] contains a table for values of Siegel coefficients $b_{l}(h)$ for $4 \leq h \leq 40$. We quote the values of Siegel coefficients which will be necessary in our computation:

$$
\begin{aligned}
b_{1}(6) & =-\frac{1}{504}, \\
b_{1}(12) & =-\frac{1}{8190}, \quad b_{2}(12)=\frac{1}{196560}, \\
b_{1}(18) & =-\frac{22}{3591}, \quad b_{2}(18)=-\frac{1}{86184} .
\end{aligned}
$$

The essence of Siegel's formula is that it transforms an infinite series (i.e., the value of a zeta function) into finite sums involving $S_{l}^{K}(2 s)$ which itself is a finite sum of powers of divisors of ideal $((\nu) \delta)$ over the $\nu$ 's in $K$ which satisfy the Siegel conditions described in (3). Therefore we need to establish the following two items to compute $S_{l}^{K}(2 s)$ :

(i) the method of computing the sum of a divisor function $\sigma_{r}(\mathfrak{A})$ for an integral ideal $\mathfrak{A}$,

(ii) the description of $\nu$ 's in $K$ which satisfy Siegel conditions described in (3).

In Section 3, we shall develop a method of computing the sum of divisor function $\sigma_{r}(\mathfrak{A})$ when $K$ is a cyclic extension of $\mathbb{Q}$ of prime degree. In Section 4 , we shall give a full description of $\nu$ 's in $K$ which satisfy Siegel conditions when $K$ is the simplest cubic field. At this moment, we examine the sum in equation (3) more closely for an arbitrary totally real algebraic number field $K$, and introduce the notion of a Siegel lattice which is first studied in [3]. 
Let $K$ be a totally real algebraic number field of degree $n$ and $S_{K}$ (or simply $S$ ) be the set of elements in $K$ which satisfy Siegel conditions described in (3). Fix an integral basis $\left\{\alpha_{1}, \ldots, \alpha_{n}\right\}$ of $K$. For $\nu \in K$, we can write

$$
\nu=x_{1} \alpha_{1}+\cdots+x_{n} \alpha_{n}, \quad x_{i} \in \mathbb{Q},
$$

and we have an embedding $\phi: K \longrightarrow \mathbb{R}^{n}$ given by

$$
\phi(\nu)=\left(x_{1}, \ldots, x_{n}\right) .
$$

The condition $\nu \in \delta^{-1}$ implies that the denominator of $x_{i}, i=1, \ldots, n$, is bounded by $D_{K}$ where $D_{K}$ denotes the discriminant of $K$. The condition $\operatorname{tr}(\nu)=l$ is equivalent to saying that $\phi(\nu)$ lies in the hyperplane

$$
a_{1} x_{1}+\cdots+a_{n} x_{n}=l,
$$

where $a_{i}=\operatorname{tr}_{K / \mathbb{Q}}\left(\alpha_{i}\right)$. Finally the condition $\nu \gg 0$ becomes $n$ distinct linear inequalities defined over $K$ in the variables $x_{1}, \ldots, x_{n}$. Therefore the elements $\nu$ in $S$ can be put in one-to-one correspondence to the lattice points in a bounded $(n-1)$ dimensional region under $\phi$. We shall call this lattice (or any set which can be put in one-to-one correspondence with this set under a suitable linear transformation) as a Siegel lattice for $K$ and denote it by $T_{K}$ (or simply $T$ ). Notice that equation (3) expresses $S_{l}^{K}(2 s)$ as a weight sum of divisor functions over a Siegel lattice. Hence the description of the Siegel lattice is of crucial importance in the computation of $S_{l}^{K}(2 s)$.

\section{Computation of the sum of Divisors}

In this section, we develop a method of computing the sum of the divisor function of $K$ when $K$ is a cyclic extension of $\mathbb{Q}$ of prime degree.

Let $K$ be a cyclic field of prime degree $q$ and $W$ denote the group of $q$ th roots of unity and $\zeta$ be a primitive $q$ th root of unity. We define an arithmetic function $\chi: \mathbb{N} \longrightarrow W \cup\{0\}$ in the following manner.

For a prime $p$, we set

$$
\chi(p)= \begin{cases}0 & \text { if } \quad p \text { is ramified in } K / \mathbb{Q}, \\ 1 & \text { if } p \text { splits completely in } K / \mathbb{Q}, \\ \zeta & \text { if } p \text { is inert in } K / \mathbb{Q}\end{cases}
$$

and extend $\chi$ multiplicatively. We put $\chi^{j}$ by $\chi_{j}$ for $j=0,1,2, \ldots, q-1$.

Lemma 3.1. Let $\zeta$ be a primitive qth root of unity. Then we have

$$
\sum_{\substack{s_{1}+\cdots+s_{q-1}=t \\ s_{1}, \ldots, s_{q-1} \geq 0}} \zeta^{s_{1}+2 s_{2}+\cdots+(q-1) s_{q-1}}= \begin{cases}1 & \text { if } t \equiv 0(\bmod q), \\ -1 & \text { if } t \equiv 1(\bmod q), \\ 0 & \text { otherwise. }\end{cases}
$$

Proof. Consider the polynomial

$$
\phi_{i}(x)=1+\zeta^{i} x+\zeta^{2 i} x^{2}+\cdots=\sum_{k=0}^{\infty} \zeta^{i k} x^{k}
$$

and put

$$
\phi(x)=\prod_{i=1}^{q-1} \phi_{i}(x)
$$


By simple computation, we have

$$
\phi(x)=\sum_{t=0}^{\infty} a_{t} x^{t}
$$

where

$$
a_{t}=\sum_{\substack{s_{1}+\cdots+s_{q-1}=t \\ s_{1}, \ldots, s_{q-1} \geq 0}} \zeta^{s_{1}+2 s_{2}+\cdots+(q-1) s_{q-1}} .
$$

Note that $\phi_{i}(x)=\frac{1}{1-\zeta^{i} x}$ and

$$
\begin{aligned}
\phi(x) & =\prod_{i=1}^{q-1} \frac{1}{1-\zeta^{i} x} \\
& =\frac{1-x}{\prod_{i=0}^{q-1}\left(1-\zeta^{i} x\right)}=\frac{1-x}{1-x^{q}}=(1-x)\left(1+x^{q}+x^{2 q}+\cdots\right), \quad|x|<1 .
\end{aligned}
$$

By comparison of coefficients, we obtain

$$
a_{t}= \begin{cases}1 & \text { if } t \equiv 0(\bmod q) \\ -1 & \text { if } t \equiv 1(\bmod q) \\ 0 & \text { otherwise }\end{cases}
$$

Theorem 3.2. Let $\mathfrak{A}$ be an integral ideal of $K$. Then, for any $r \geq 0$,

$$
\sigma_{r}(\mathfrak{A})=\sum_{\left(j_{1} \cdots j_{q-1}\right)^{2} \mid \mathfrak{A} q} \chi_{1}\left(j_{1}\right) \cdots \chi_{q-1}\left(j_{q-1}\right) j_{1}^{r} \cdots j_{q-1}^{r} \sigma_{r}\left(\frac{N}{j_{1}^{2} \cdots j_{q-1}^{2}}\right),
$$

where $N=\operatorname{Norm}_{K / \mathbb{Q}}(\mathfrak{A})$ denotes the norm of $\mathfrak{A}$, the function $\sigma_{r}$ on the right-hand side is the usual sum of divisors function defined by equation (2) in Section 2, and the summation is over all positive integers $j_{1}, \ldots, j_{q-1}$ such that $\left(j_{1} \cdots j_{q-1}\right)^{2}$ divides $\mathfrak{A}^{q}$, i.e., $\left(\left(j_{1} \cdots j_{q-1}\right)^{2}\right) \supset \mathfrak{A}^{q}$.

Proof. We put $\widetilde{\sigma_{r}}(\mathfrak{A})$ to be the right-hand side of $(15)$. Since $\sigma_{r}(\mathfrak{A})$ and $\widetilde{\sigma_{r}}(\mathfrak{A})$ are both multiplicative, we may assume that $\mathfrak{A}$ is a power $\mathfrak{P}^{m}$ of a prime ideal $\mathfrak{P}$. Let $p$ be the rational prime lying below $\mathfrak{P}$. Then

$$
N(\mathfrak{P})=p^{f},
$$

where $f$ is the inertial degree of $\mathfrak{P}$ in $K / \mathbb{Q}$. We have

$$
\sigma_{r}(\mathfrak{A})=\sigma_{r}\left(\mathfrak{P}^{m}\right)=\sum_{s=0}^{m} N\left(\mathfrak{P}^{s}\right)^{r}=\sum_{s=0}^{m} p^{f s r}=\sigma_{f r}\left(p^{m}\right) .
$$

To evaluate $\widetilde{\sigma_{r}}(\mathfrak{A})$, we must distinguish three cases, according to the value of $\chi(p)$. Case 1. $\chi(p)=1$. Since $[K: \mathbb{Q}]=q$ is a prime, $(p)$ splits completely in $K / \mathbb{Q}$, and $f=1$. Write

$$
(p)=\mathfrak{P}_{1} \ldots \mathfrak{P}_{q},
$$

where $\mathfrak{P}_{1}=\mathfrak{P}$ and $\mathfrak{P}_{i}, i=2, \ldots, q$, are the conjugates of $\mathfrak{P}$. If $j \mid \mathfrak{A}^{q}$, then $j \mid \mathfrak{P}_{i}^{m q}$ for each $i$. So,

$$
j^{q} \mid p^{m q}
$$


This means that $j$ is a power of $p$. Since $p$ splits completely, we must have $j=1$. Hence $j_{1}=\cdots=j_{q-1}=1$ is the only term on the right-hand side of (16). Therefore we have

$$
\widetilde{\sigma_{r}}(\mathfrak{A})=\widetilde{\sigma_{r}}\left(\mathfrak{P}^{m}\right)=\sigma_{r}(N)=\sigma_{r}\left(p^{m}\right),
$$

and this coincides with (16) since $f=1$.

Case 2. $\chi(p)=0$. Since $p$ is ramified in $K / \mathbb{Q},(p)=\mathfrak{P}^{q}$ and $f=1$. If $j \mid \mathfrak{A}$, then $j \mid \mathfrak{P}^{m}$. So $j^{q} \mid p^{m}$, which implies that $j$ is a power of $p$. Since $\chi(p)=0$, the only term in $\widetilde{\sigma_{r}}(\mathfrak{A})$ that does not vanish is the term corresponding to $j_{1}=\cdots=j_{q-1}=1$, namely, $\sigma_{r}(N)$. Therefore, we have

$$
\widetilde{\sigma_{r}}(\mathfrak{A})=\sigma_{r}(N)=\sigma_{r}\left(p^{m}\right),
$$

and this coincides with (16) since $f=1$.

Case 3. $\chi(p)=\zeta$, a primitive $q$ th root of unity. Since $p$ is inert in $K / \mathbb{Q}, \mathfrak{P}=(p)$, and $f=q$. By definition,

$$
\widetilde{\sigma_{r}}(\mathfrak{A})=\widetilde{\sigma_{r}}\left(\left(p^{m}\right)\right)=\sum_{\left(j_{1} \cdots j_{q-1}\right)^{2} \mid p^{m q}} \chi_{1}\left(j_{1}\right) \cdots \chi_{q-1}\left(j_{q-1}\right) j_{1}^{r} \cdots j_{q-1}^{r} \sigma_{r}\left(\frac{N}{j_{1}^{2} \cdots j_{q-1}^{2}}\right) .
$$

Write $j_{i}=p^{s_{i}}, i=1, \ldots, q-1$. Then (17) becomes

$$
\widetilde{\sigma_{r}}(\mathfrak{A})=\sum_{\substack{2\left(s_{1}+\cdots+s_{q-1}\right) \leq m q \\ s_{i} \geq 0}} p^{r\left(s_{1}+\cdots+s_{q-1}\right)} \zeta^{s_{1}} \zeta^{2 s_{2}} \cdots \zeta^{(q-1) s_{q-1}} \sigma_{r}\left(p^{m q-2\left(s_{1}+\cdots+s_{q-1}\right)}\right) .
$$

Furthermore, from (18) it follows that

$$
\widetilde{\sigma_{r}}(\mathfrak{A})=\sum_{t=0}^{\left[\frac{m q}{2}\right]} \sum_{\substack{s_{1}+\cdots+s_{q-1}=t \\ s_{i} \geq 0}} p^{r t} \sigma_{r}\left(p^{m q-2 t}\right) \zeta^{s_{1}+2 s_{2}+\cdots+(q-1) s_{q-1}} .
$$

Finally, we get

$$
\widetilde{\sigma_{r}}(\mathfrak{A})=\sum_{t=0}^{\left[\frac{m q}{2}\right]} p^{r t} \sigma_{r}\left(p^{m q-2 t}\right) \sum_{\substack{s_{1}+\cdots+s_{q-1}=t \\ s_{i} \geq 0}} \zeta^{s_{1}+2 s_{2}+\cdots+(q-1) s_{q-1}} .
$$

Now we consider two cases, say $m$ is even or $m$ is odd. We only give a proof for the case that $m$ is even since the other case can be treated similarly. Write $m=2 m^{\prime}$. Then $\left[\frac{m q}{2}\right]=m^{\prime} q$. By Lemma 3.1. (20) becomes

$$
\begin{aligned}
\widetilde{\sigma_{r}}(\mathfrak{A}) & =\sum_{t=0}^{m^{\prime} q} p^{r t} \sigma_{r}\left(p^{2 m^{\prime} q-2 t}\right) a_{t} \\
& =\sum_{\substack{t=q t^{\prime} \\
0 \leq t^{\prime} \leq m^{\prime}}} \frac{\left(p^{r}\right)^{2 m^{\prime} q-q t^{\prime}+1}-\left(p^{r}\right)^{q t^{\prime}}}{p^{r}-1}-\sum_{\substack{t=q t^{\prime}+1 \\
0 \leq t^{\prime} \leq m^{\prime}-1}} \frac{\left(p^{r}\right)^{2 m^{\prime} q-q t^{\prime}}-\left(p^{r}\right)^{q t^{\prime}+1}}{p^{r}-1} .
\end{aligned}
$$

So, we get

$$
\widetilde{\sigma_{r}}(\mathfrak{A})=\frac{1}{p^{r}-1}\left\{\sum_{l=0}^{2 m^{\prime}}\left(p^{r}\right)^{q l+1}-\sum_{l=0}^{2 m^{\prime}}\left(p^{r}\right)^{q l}\right\}=\sigma_{r q}\left(p^{m}\right) .
$$


This agrees with (16) since $f=q$.

Remark 3.1. When $q=2$, i.e., $K$ is a real quadratic field, the equation (15) becomes the formula obtained by Zagier [10].

\section{Description of a Siegel lattice for the Simplest Cubic fields}

In this section, we shall give a full description of a Siegel lattice for the simplest cubic field. As a result, we derive a formula for the number of points in a Siegel lattice.

Let $m(\geq-1)$ be an integer such that $m^{2}+3 m+9$ is square-free and $K$ be the simplest cubic field defined by the irreducible polynomial

$$
f(x)=x^{3}+m x^{2}-(m+3) x+1 .
$$

Recall that the discriminant $d_{K}$, the ring of integers $\mathcal{O}_{K}$, and the different $\delta_{K}$ of $K$ are given, respectively, by

$$
\begin{array}{r}
d_{K}=D^{2}=\left(m^{2}+3 m+9\right)^{2}, \\
\mathcal{O}_{K}=\mathbb{Z}[\rho]=\mathbb{Z} \bigoplus \mathbb{Z} \rho \bigoplus \mathbb{Z} \rho^{2}, \\
\delta_{K}=\left(f^{\prime}(\rho)\right)=\left(-(m+3)+2 m \rho+3 \rho^{2}\right),
\end{array}
$$

where $\rho$ denotes the negative root of $f(x)$. Let $\nu$ be an element of $K$. We can write

$$
\nu=\alpha+\beta \rho+\gamma \rho^{2}, \quad \alpha, \beta, \gamma \in \mathbb{Q} .
$$

Now suppose that $\nu$ satisfies Siegel conditions, i.e.,

$$
\nu \in \delta^{-1}, \quad \nu \gg 0, \quad \operatorname{tr}(\nu)=l .
$$

1. $\nu \in \delta^{-1}$

$$
\nu \in \delta^{-1} \Longleftrightarrow \nu\left(-(m+3)+2 m \rho+3 \rho^{2}\right) \in \mathcal{O}_{K} .
$$

Hence we can write

$$
\nu\left(-(m+3)+2 m \rho+3 \rho^{2}\right)=A+B \rho+C \rho^{2},
$$

with $A, B, C \in \mathbb{Z}$.

From $(25),(27)$, we obtain the following system of linear equations:

$$
\begin{aligned}
-(m+3) \alpha-3 \beta+m \gamma & =A, \\
2 m \alpha+2(m+3) \beta+\left(-m^{2}-3 m-3\right) \gamma & =B, \\
3 \alpha-m \beta+\left(m^{2}+2 m+6\right) \gamma & =C .
\end{aligned}
$$

Using Cramer's rule, it follows that

$$
\alpha=\frac{a}{D}, \quad \beta=\frac{b}{D}, \quad \gamma=\frac{c}{D}, \quad(a, b, c) \in \Lambda,
$$

where $\Lambda$ is a free module of rank 3 in $\mathbb{Z}^{3}$ and $D=m^{2}+3 m+9$.

By substitution of (31) into (28),(29),(30), we finally have

$$
\begin{aligned}
-(m+3) a-3 b+m c & =D A \equiv 0 & & (\bmod D), \\
2 m a+2(m+3) b-\left(m^{2}+3 m+3\right) c & =D B \equiv 0 & & (\bmod D), \\
3 a-m b+\left(m^{2}+2 m+6\right) c & =D C \equiv 0 & & (\bmod D) .
\end{aligned}
$$

2. $\operatorname{tr}(\nu)=l$

$$
\operatorname{tr}(\nu)=l \Longleftrightarrow 3 \alpha-m \beta+\left(m^{2}+2 m+6\right) \gamma=l .
$$


From (31),(34), it follows that

$$
C=l, b=\frac{3 a+\left(m^{2}+2 m+6\right) c-l D}{m} .
$$

By substitution of (35) into (32), we have

$$
-a+3 l-2 c=m A .
$$

In particular, $m$ divides $a+2 c-3 l$. Now we introduce a new variable $t$ by the formula

$$
t=\frac{a+2 c-3 l}{m} .
$$

By substitution of (37) into (35), we have

$$
b=3 t+(m+2) c-l(m+3) .
$$

3. $\nu \gg 0$

$$
\nu \gg 0 \Longleftrightarrow D \nu=a+b \rho+c \rho^{2} \gg 0 .
$$

This condition becomes three linear inequalities in the variables $a, b, c$ defined over $K$. Using (35),(37), we have the following system of linear inequalities in the variables $c, t$ defined over $K$ :

$$
\begin{aligned}
\left(\rho^{2}+(m+2) \rho-2\right) c+(m+3 \rho) t+l(3-(m+3) \rho) & >0, \\
\left(\rho^{\prime 2}+(m+2) \rho^{\prime}-2\right) c+\left(m+3 \rho^{\prime}\right) t+l\left(3-(m+3) \rho^{\prime}\right) & >0, \\
\left(\rho^{\prime \prime 2}+(m+2) \rho^{\prime \prime}-2\right) c+\left(m+3 \rho^{\prime \prime}\right) t+l\left(3-(m+3) \rho^{\prime \prime}\right) & >0 .
\end{aligned}
$$

Let $L_{1}$ (resp., $\left.L_{2}, L_{3}\right)$ denote the line in $(c, t)$-plane defined by the left-hand side of (38) (resp., (39),(40)). By simple computation, we obtain

$$
\begin{aligned}
& \left(\left(-\rho+\rho^{\prime}\right) l, \frac{l}{\rho^{\prime \prime}}\right) \quad \text { is the point of intersection of } \quad L_{1}=L_{2}=0 \\
& \left(\left(-\rho^{\prime}+\rho^{\prime \prime}\right) l, \frac{l}{\rho}\right) \quad \text { is the point of intersection of } \quad L_{2}=L_{3}=0 \\
& \left(\left(-\rho^{\prime \prime}+\rho\right) l, \frac{l}{\rho^{\prime}}\right) \quad \text { is the point of intersection of } \quad L_{3}=L_{1}=0
\end{aligned}
$$

We summarize the above computation as in the following proposition.

Proposition 4.1. Let $m(\geq-1)$ be an integer such that $D=m^{2}+3 m+9$ is squarefree, and $K$ be the simplest cubic field defined by equation (21). Let $S$ be the set of elements in $K$ which satisfy Siegel conditions described by equation (26) and $T$ be the set of integral points in $(c, t)$-plane which lie inside of the triangle surrounded by the lines $L_{1}=0, L_{2}=0$, and $L_{3}=0$. For $\nu \in S$, by equation (31), we can write

$$
\nu=\frac{a}{D}+\frac{b}{D} \rho+\frac{c}{D} \rho^{2}, \quad a, b, c \in \mathbb{Z} .
$$

Then the mapping $\eta: S \longrightarrow T$ given by $\eta(\nu)=(c, t)$, where

$$
c=c, t=\frac{a+2 c-3 l}{m}
$$


gives a one-to-one correspondence between $S$ and $T$. The inverse mapping $\tau: T \longrightarrow$ $S$ is given by

$$
\tau(c, t)=\nu=\frac{a}{D}+\frac{b}{D} \rho+\frac{c}{D} \rho^{2},
$$

where $a=m t-2 c+3 l, b=3 t+(m+2) c-l(m+3)$.

Remark 4.1. A straightforward calculation shows that $\nu=\tau(c, t)$ satisfies equation (27) with $A, B, C$ in $\mathbb{Z}$.

Example 4.1. As an illustration of our discussion, we describe the Siegel lattice $T$ for the simplest cubic field $K$ with $m=8$. We first note that $g_{l}(x)=x^{3}-l^{2} D x+l^{3} D$ (resp., $\left.h_{l}(x)=x^{3}-l(m+3) x^{2}+m l^{2} x+l^{3}\right)$ is the cubic polynomial whose roots are the conjugates of $\left(-\rho+\rho^{\prime}\right) l$ (resp., $\left.\frac{l}{\rho}\right)$. By estimating the roots of $g_{l}(x)$ and $h_{l}(x)$, we can find the rough location of vertices of the triangle. For the roots of $g_{l}(x)$, we have

$$
\begin{aligned}
(-m-3) l & <\left(-\rho^{\prime \prime}+\rho\right) l<(-m-2) l \\
& <\left(-\rho^{\prime}+\rho^{\prime \prime}\right) l<(m+1) l<\left(-\rho+\rho^{\prime}\right) l<(m+2) l .
\end{aligned}
$$

Similarly, we obtain

$$
-l<\frac{l}{\rho}<0<\frac{l}{\rho^{\prime \prime}}<l \leq(m+2) l<\frac{l}{\rho^{\prime}}<(m+3) l .
$$

For $(c, t) \in T$, the corresponding $\nu$ in $S$ is given by

$$
\nu=\frac{a}{D}+\frac{b}{D} \rho+\frac{c}{D} \rho^{2}
$$

where $a=m t-2 c+3 l, b=3 t+(m+2) c-(m+3) l, c=c$. Since

$$
\delta=\left(m+3-2 m \rho-3 \rho^{2}\right),
$$

it follows from a simple computation that

$$
(\nu) \delta=\left(t+(-2 t-c+2 l) \rho-l \rho^{2}\right),
$$

where $b=2 t+c-2 l$. Let $N(c, t)$ denote the norm function $N_{K / \mathbb{Q}}((\nu) \delta)$. By elementary computation, we obtain

$$
\begin{aligned}
N(c, t)= & {\left[l t^{2}+(c-l) l t\right] m^{2} } \\
& +\left[-2 t^{3}+(-3 c+6 l) t^{2}+\left(-c^{2}+3 l c\right) t+\left(-l c^{2}+3 l^{2} c-2 l^{3}\right)\right] m \\
& +\left[-3 t^{3}+\left(3 c^{2}-9 l c+9 l^{2}\right) t+\left(c^{3}-6 l c^{2}+9 l^{2} c-3 l^{3}\right)\right] .
\end{aligned}
$$

Note that a point $(c, t)$ in the plane near the boundary the triangle lies inside the triangle if and only if $N(c, t)>0$. Combining these data, we conclude that the Siegel lattice for $K$ is given as in Figures 1 and 2 .

We now describe the Galois action on a Siegel lattice. We start from the following simple observation.

Lemma 4.2. Let $K$ be a totally real Galois extension of $\mathbb{Q}$ with Galois group $G$. If $\nu \in K$ satisfies the Siegel conditions described in equation (26), then so does $\sigma(\nu)$ for $\sigma \in G$.

Proof. This is clear! The most important thing is to realize that this is an important fact. 


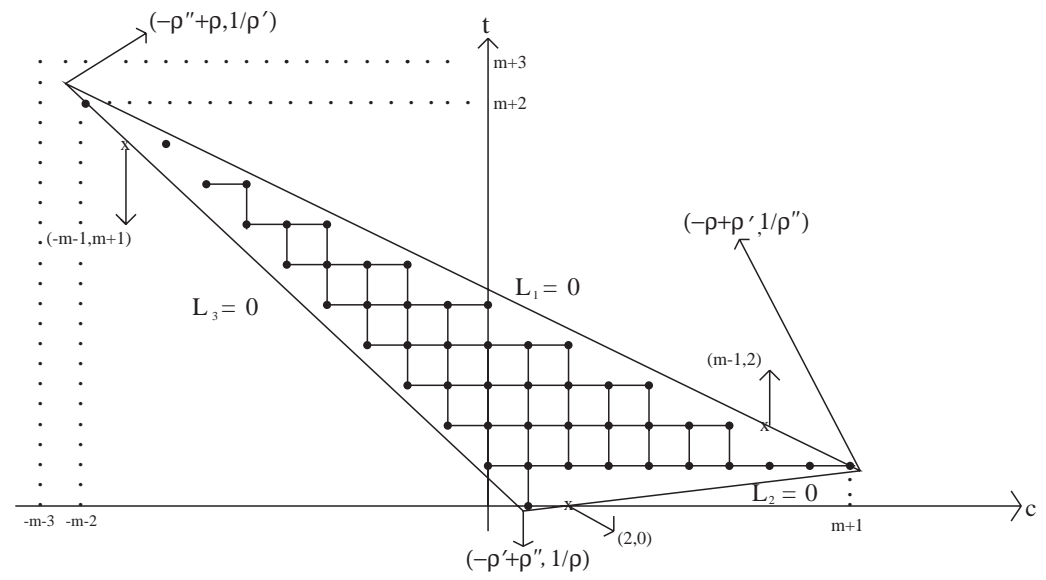

- : point which belongs to the triangle

$\mathrm{x}$ : point which does not belong to the triangle

FIgURE 1. Siegel lattice for $m=8$ with $\operatorname{tr}(\nu)=1$.

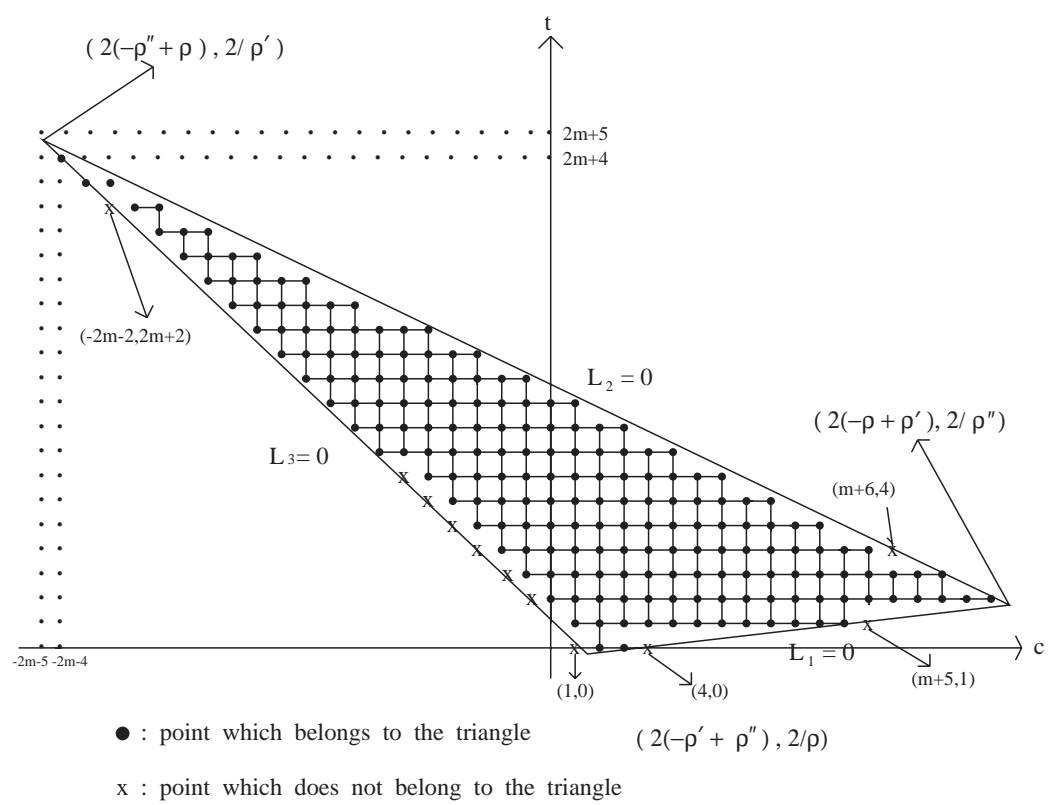

Figure 2. Siegel lattice for $m=8$ with $\operatorname{tr}(\nu)=2$.

By Lemma 4.2, the Galois group $G=\operatorname{Gal}(K / \mathbb{Q})$ acts on the set $S$ and $S$ can be put into one-to-one correspondence with the Siegel lattice $T$. Therefore, we have the induced Galois action on $T$. Now we return to the simplest cubic field case and describe the Galois action on $T$.

Proposition 4.3 (Galois action on a Siegel lattice). Let $m(\geq-1)$ be an integer such that $D=m^{2}+3 m+9$ is square-free, and let $K$ be the simplest cubic field 
defined by equation (21). Then the Galois group $G(=\langle\sigma\rangle)$ induces an action on $T$ given by

$$
\sigma(c, t)=(-2 c-3 t+(m+3) l, c+t) .
$$

If $l$ is not divisible by 3 , then every $G$-orbit contains three points. In particular, $N_{l}$ is divisible by 3 , where $N_{l}$ denotes the number of lattice points in $T$ which corresponds to $\operatorname{tr}(\nu)=l$.

Proof. Let $(c, t) \in T$. By Proposition 4.1, it corresponds to $\nu \in S$ where $\nu$ is given by

$$
D \nu=(m t-2 c+3 l)+\{3 t+(m+2) c-(m+3) l\} \rho+c \rho^{2} .
$$

By an actual computation, we obtain

$$
\begin{aligned}
D \nu^{\prime}= & \{(m+6) t+(m+4) c-(2 m+3) l\} \\
& +\left\{-3(m+1) t-(2 m+1) c+\left(m^{2}+4 m+3\right) l\right\} \rho \\
& +\{-3 t-2 c+(m+3) l\} \rho^{2} .
\end{aligned}
$$

From the transformation formula (45), it follows that $\eta\left(\nu^{\prime}\right)=\left(c^{\prime}, t^{\prime}\right)$, where $c^{\prime}=$ $-2 c-3 t+(m+3) l$ and $t^{\prime}=c+t$. This proves the first assertion. Now suppose that the Galois action on $T$ has a fixed point, say $(c, t)$. Then it follows from (49) that

$$
(c, t)=(-2 c-3 t+(m+3) l, c+t) .
$$

Thus we must have $c=0$ and $(m+3) l=3 t$. Since $m$ is not divisible by 3 , we conclude that $l$ is divisible by 3 .

We now prove the main result of this section.

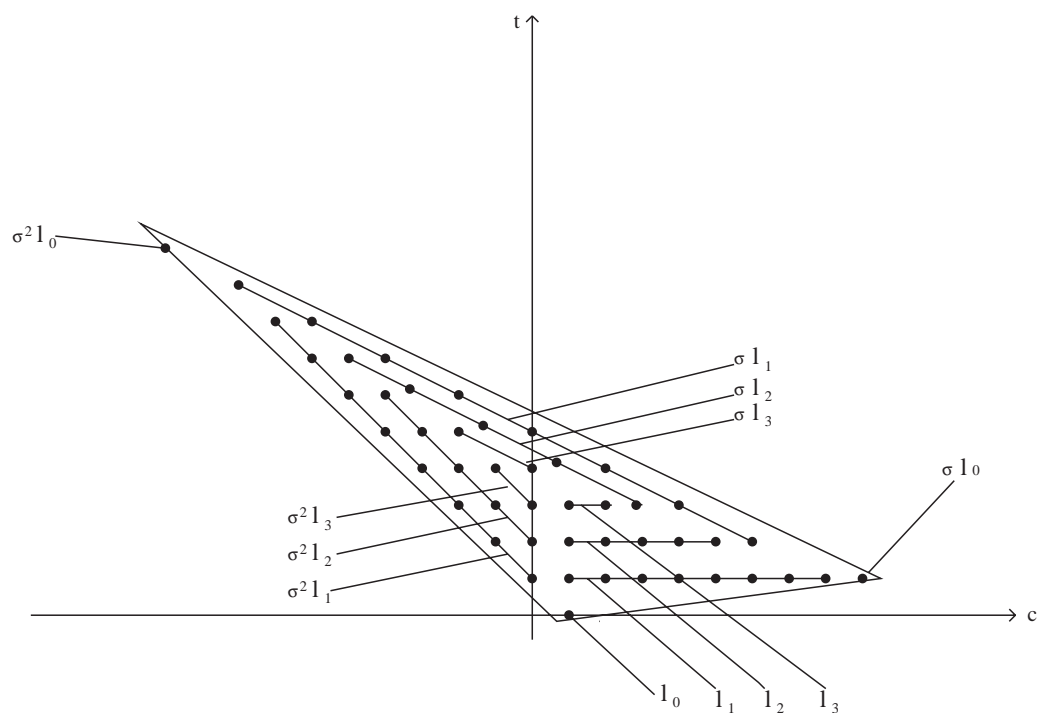

Figure 3. Galois action on the Siegel lattice for $m=8$ with $\operatorname{tr}(\nu)=1$. 
Theorem 4.4. Let $m(\geq-1)$ be an integer such that $D=m^{2}+3 m+9$ is squarefree, and let $K$ be the simplest cubic field defined by (21). Let $N_{l}$ denote the number of Siegel lattice points for $K$ which corresponds to $\operatorname{tr}(\nu)=l$. Then we have

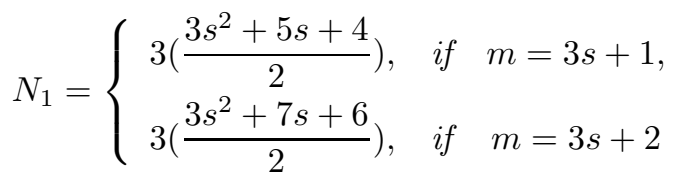

and

$$
N_{2}=\left\{\begin{array}{cl}
3\left(6 s^{2}+10 s+9\right), & \text { if } \quad m=3 s+1, \\
3\left(6 s^{2}+14 s+13\right), & \text { if } \quad m=3 s+2 .
\end{array}\right.
$$

Proof. We only give a detailed proof for the case $m=3 s+2$, since the other case can be treated in the same manner. We assume that $m \geq 5$. (The case of $m=2$ can be treated by direct computation.) The basic idea of the proof is to find a set of representatives of "good shape" for the Galois action on $T$.

First, we consider the case of $\operatorname{tr}(\nu)=1$. First note that $(1,0)$ is the only point in the Siegel lattice with $t=0$. Let $l_{0}$ be the point $(1,0)$. Then $\sigma\left(l_{0}\right)$ (resp., $\left.\sigma^{2}\left(l_{0}\right)\right)$ is the point $(m+1,1)$ (resp., $(-m-2, m+1))$. For $1 \leq i \leq s+1$, let $l_{i}$ be the line joining $(3 s+5-3 i, i)$ and $(1, i)$. By simple computation, we know that $\sigma\left(l_{i}\right)$ is the line joining $(-3 s-5+3 i, 3 s+5-2 i)$ and $(3 s+2-3 i, 1+i)$, and $\sigma^{2}\left(l_{i}\right)$ is the line joining $(0, i)$ and $(-3 s-2+3 i, 3 s+3-2 i)$ (see Figure 3$)$. This proves that the set of lattice points on $\bigcup_{i=0}^{s+1} l_{i}$ becomes a set of representatives for the Galois action (see Figure 5). Therefore,

$$
N_{1}=3\left\{1+\sum_{i=1}^{s+1}(3 s+5-3 i)\right\}=3\left(\frac{3 s^{2}+7 s+6}{2}\right) \text {. }
$$

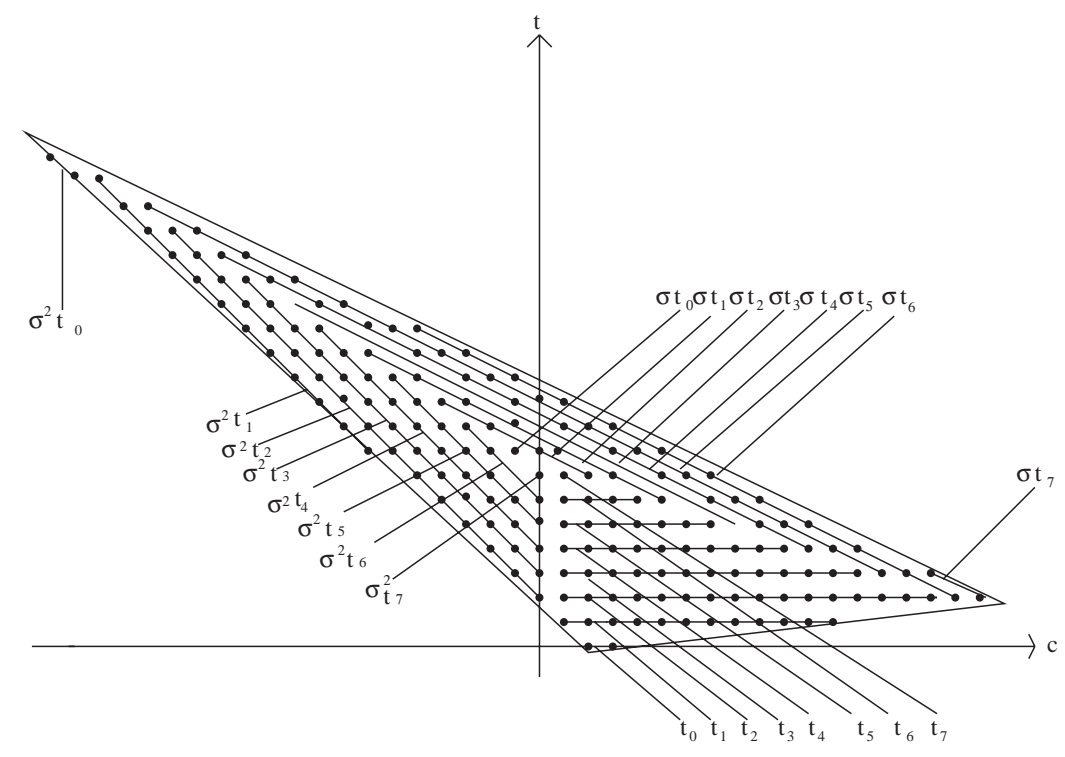

Figure 4. Galois action on the Siegel lattice for $m=8$ with $\operatorname{tr}(\nu)=2$. 


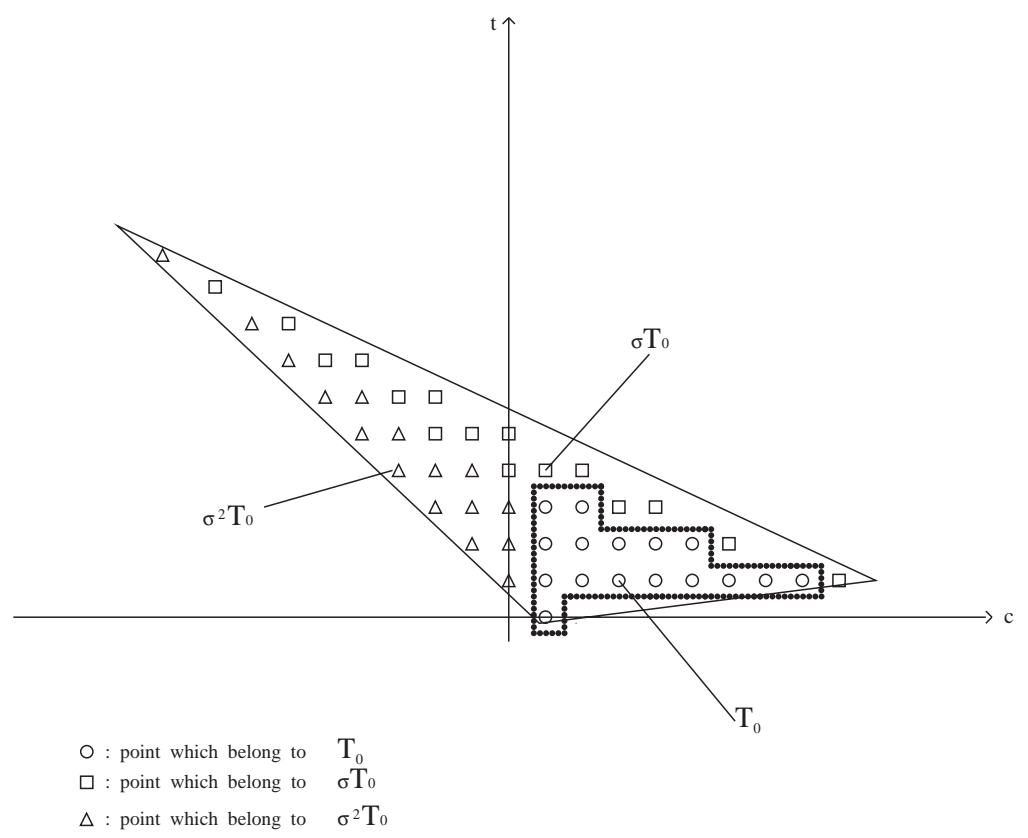

Figure 5. A set of representatives for the Galois action on the Siegel lattice for $m=8$ with $\operatorname{tr}(\nu)=1$.

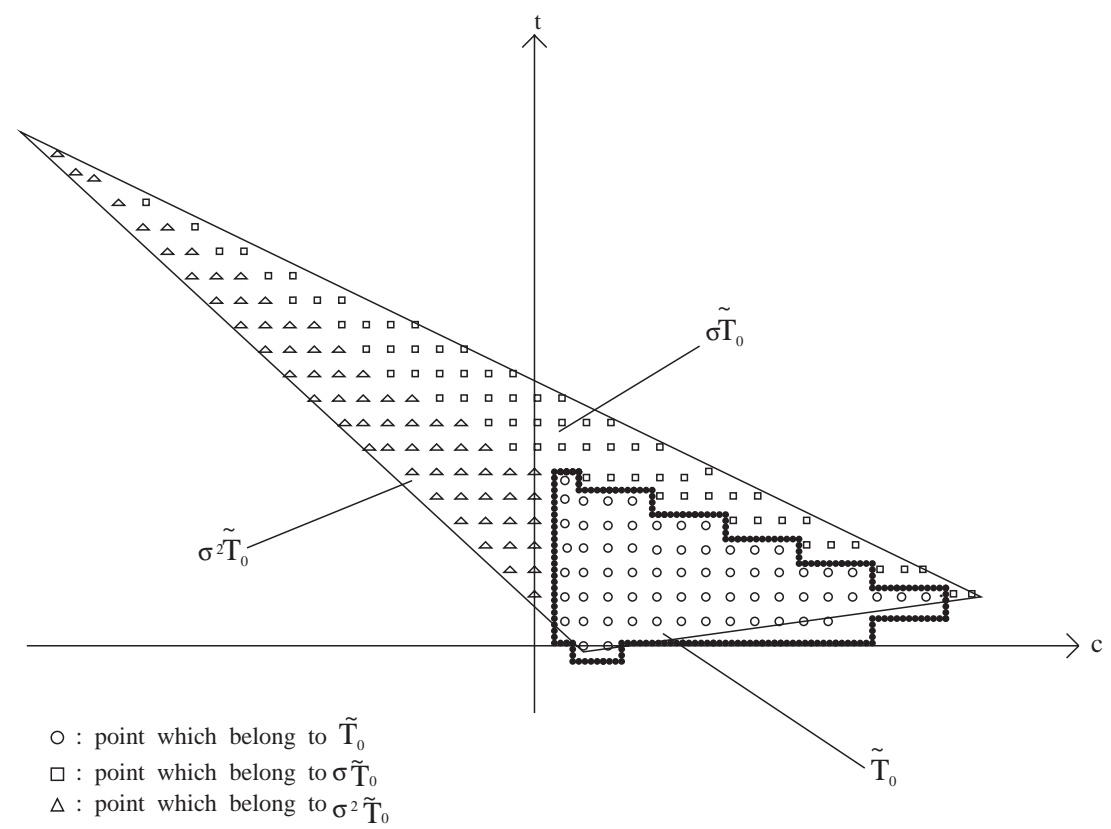

Figure 6. A set of representatives for the Galois action on the Siegel lattice for $m=8$ with $\operatorname{tr}(\nu)=2$. 
Secondly, we consider the case of $\operatorname{tr}(\nu)=2$. Let $t_{0}$ be the line joining $(2,0)$ and $(3,0)$ and $t_{1}$ be the line joining $(1,1)$ to $(3 s+6,1)$. For $2 \leq i \leq 2 s+3$, let $t_{i}$ be the line joining $(6 s+10-3 i, i)$ and $(1, i)$. As in the case of $\operatorname{tr}(\nu)=1$, the lattice

points on $\bigcup_{i=0}^{2 s+3} t_{i}$ becomes a set of representatives for the Galois action on $T$ (see Figures 4 and 6 ). Therefore,

$$
N_{2}=3\left(2+3 s+6+\sum_{i=2}^{2 s+3}(6 s+10-3 i)\right)=3\left(6 s^{2}+14 s+13\right) .
$$

\section{VAlues OF The ZETA FUnCtions}

In this section, we apply the previously discussed result to the evaluation of the zeta function of the simplest cubic field. We shall derive explicit expressions for $\zeta_{K}(-1), \zeta_{K}(-3), \zeta_{K}(-5)$ which are elementary in the sense that they involve only rational integers and not algebraic numbers or ideals. As an illustration, we present Table 1 for values of $-21 \zeta_{K}(-1), 8190 \zeta_{K}(-3)$, and $-3591 \zeta_{K}(-5)$ for the first one hundred simplest cubic fields.

Recall the definition of $S_{l}^{K}(2 s)$ :

$$
S_{l}^{K}(2 s)=\sum_{\substack{\nu \in \delta-1 \\ \nu \gg 0 \\ \operatorname{tr}(\nu)=l}} \sigma_{2 s-1}((\nu) \delta)
$$

By virtue of Theorem 3.2 we have

$$
\sigma_{2 s-1}((\nu) \delta)=\sum_{\left(j j^{\prime}\right)^{2} \mid((\nu) \delta)^{3}} \chi(j) \overline{\chi\left(j^{\prime}\right)}\left(j j^{\prime}\right)^{2 s-1} \sigma_{2 s-1}\left(\frac{N}{\left(j j^{\prime}\right)^{2}}\right),
$$

where $N$ denotes the norm of ideal $(\nu) \delta$.

Lemma 5.1. Let $p$ be a prime or $p=1$ such that $p^{2} \mid((\nu) \delta)^{3}$. Then only $p$ 's such that $p \mid(\nu) \delta$ contribute in the sum (51).

Proof. Suppose that $p^{2} \mid((\nu) \delta)^{3}$. If $p$ is inert in $K / \mathbb{Q}$, then $p \mid(\nu) \delta$. If $p$ splits in $K / \mathbb{Q}$, we can write $(p)=\mathfrak{P P}^{\prime} \mathfrak{P}^{\prime \prime}$, and

$$
(\nu) \delta=\mathfrak{P}^{a} \mathfrak{P}^{\prime}{ }^{\prime \prime} \mathfrak{P}^{\prime c} \prod \mathfrak{B}_{i},
$$

where $\left(\mathfrak{B}_{i}, p\right)=1$. Since $p^{2} \mid((\nu) \delta)^{3}$, it follows that $\min (3 a, 3 b, 3 c) \geq 2$, and consequently we have $p \mid(\nu) \delta$. Finally, if $p$ is ramified in $K / \mathbb{Q}, p$ does not contribute in the sum (51) since the character value on the right-hand side of (51) vanishes.

From the unique factorization of ideals into prime ideals, it follows that

$$
\sigma_{2 s-1}((\nu) \delta)=\sigma_{2 s-1}\left(\left(\nu^{\prime}\right) \delta\right), \quad \text { for every } \quad \nu \in \delta^{-1}
$$

Now we shall compute $S_{1}^{K}(2 s)$. Let $T$ be the Siegel lattice of $K$ which is computed in Section 4 and corresponds to $\operatorname{tr}(\nu)=l=1$. In Section 4 we have a one-to-one correspondence between points $(c, t)$ in $T$ and ideals $(\nu) \delta$, where $\nu$ is an element of $K$ which satisfies the Siegel conditions, $\nu \in \delta^{-1}, \nu \gg 0$ and $\operatorname{tr}(\nu)=1$. For $(c, t) \in T$, by equation (47), the corresponding ideal is given by

$$
(\nu) \delta=\left(t+(-2 t-c+2) \rho-\rho^{2}\right)
$$


Let $f_{m}(c, t)$ be the norm of the ideal $(\nu) \delta$. By $(48)$, it can be explicitly expressed by

$$
\begin{aligned}
f_{m}(c, t)= & {\left[t^{2}+(c-1) t\right] m^{2} } \\
& +\left[-2 t^{3}+(-3 c+6) t^{2}+\left(-c^{2}+3 c\right) t+\left(-c^{2}+3 c-2\right)\right] m \\
& +\left[-3 t^{3}+\left(3 c^{2}-9 c+9\right) t+\left(c^{3}-6 c^{2}+9 c-3\right)\right] .
\end{aligned}
$$

Note that $p \mid(\nu) \delta$ if and only if $p=1$. By Lemma 5.1 we have

$$
\sigma_{2 s-1}((\nu) \delta)=\sigma_{2 s-1}\left(f_{m}(c, t)\right) .
$$

Thus we have

$$
S_{1}^{K}(2 s)=\sum_{(c, t) \in T} \sigma_{2 s-1}\left(f_{m}(c, t)\right)=3 \sum_{(c, t) \in T_{0}} \sigma_{2 s-1}\left(f_{m}(c, t)\right),
$$

where $T_{0}$ denotes a set of representatives for the Galois action described in the proof of Theorem 4.4

Next we shall compute $S_{2}^{K}(2 s)$. Let $T$ denote the Siegel lattice which corresponds to $\operatorname{tr}(\nu)=l=2$. As in the case $l=1$, for $(c, t) \in T$ the corresponding ideal is given by

$$
(\nu) \delta=\left(t+(-2 t-c+4) \rho-2 \rho^{2}\right) .
$$

Therefore $p \mid(\nu) \delta$ if and only if $p=1$ when $c$ or $t$ is odd, and $p \mid(\nu) \delta$ if and only if $p=1$ or $p=2$ when both $c$ and $t$ are even. Let $g_{m}(c, t)$ denote the norm of the ideal in (56), which is given explicitly by

$$
\begin{aligned}
g_{m}(c, t)= & {\left[2 t^{2}+2(c-2) t\right] m^{2} } \\
& +\left[-2 t^{3}+(-3 c+12) t^{2}+\left(-c^{2}+6 c\right) t+\left(-2 c^{2}+12 c-16\right)\right] m \\
& +\left[-3 t^{3}+\left(3 c^{2}-18 c+36\right) t+\left(c^{3}-12 c^{2}+36 c-24\right)\right] .
\end{aligned}
$$

If either $c$ or $t$ is odd, then

$$
\sigma_{2 s-1}((\nu) \delta)=\sigma_{2 s-1}\left(g_{m}(c, t)\right) .
$$

If both $c$ and $t$ are even, then

$$
\sigma_{2 s-1}((\nu) \delta)=\sigma_{2 s-1}\left(g_{m}(c, t)\right)+[\chi(2)+\bar{\chi}(2)] 2^{2 s-1} \sigma_{2 s-1}\left(\frac{g_{m}(c, t)}{4}\right) .
$$

Since $f_{m}(x)$ is irreducible over $G F(2), 2$ is inert in $K / \mathbb{Q}$. Hence $\chi(2)+\bar{\chi}(2)=-1$ by definition of $\chi$. Therefore we have

$$
S_{2}^{K}(2 s)=3\left[\sum_{(c, t) \in \tilde{T}_{0}} \sigma_{2 s-1}\left(g_{m}(c, t)\right)-2^{2 s-1} \sum_{\substack{(c, t) \in \tilde{T}_{0} \\ c, t: \text { even }}} \sigma_{2 m-1}\left(\frac{g_{m}(c, t)}{4}\right)\right],
$$

where $\tilde{T}_{0}$ denotes a set of representatives for the Galois action. By (6), (7), (8), (55), and (58), we finally have the following theorem.

Theorem 5.2. Let $m(\geq-1)$ be an integer such that $D=m^{2}+3 m+9$ is squarefree, and let $K$ be the simplest cubic field defined by equation (21) in Section 4 . 
Define elementary functions $f_{m}(c, t)$ and $g_{m}(c, t)$ by (54),(57), respectively. Then we have

$$
\begin{aligned}
-21 \zeta_{K}(-1)= & \sum_{(c, t) \in T_{0}} \sigma_{1}\left(f_{m}(c, t)\right), \\
8190 \zeta_{K}(-3)= & -24 * \sum_{(c, t) \in T_{0}} \sigma_{3}\left(f_{m}(c, t)\right) \\
& +\sum_{(c, t) \in \tilde{T}_{0}} \sigma_{3}\left(g_{m}(c, t)\right)-8 * \sum_{\substack{(c, t) \in \tilde{T}_{0} \\
c, t: e v e n}} \sigma_{3}\left(\frac{g_{m}(c, t)}{4}\right), \\
-3591 \zeta_{K}(-5)= & 528 * \sum_{(c, t) \in T_{0}} \sigma_{5}\left(f_{m}(c, t)\right) \\
& +\sum_{(c, t) \in \tilde{T}_{0}} \sigma_{5}\left(g_{m}(c, t)\right)-32 * \sum_{\substack{(c, t) \in \tilde{T}_{0} \\
c, t: \text { :even }}} \sigma_{5}\left(\frac{g_{m}(c, t)}{4}\right),
\end{aligned}
$$

where $T_{0}$ (resp., $\tilde{T}_{0}$ ) denotes a set of representatives for the Galois action on the Siegel lattice for $\operatorname{tr}(\nu)=l=1$ (resp., $\operatorname{tr}(\nu)=l=2$ ).

From Theorem 5.2, we can easily compute values of the zeta function of the simplest cubic field. As an illustration, we give Table 1 for values of $-21 \zeta_{K}(-1)$, $8190 \zeta_{K}(-3)$, and $-3591 \zeta_{K}(-5)$ for the first one hundred simplest cubic fields.

We first give some remarks on our computation.

Remark 5.1. Halbritter and Pohst 2] developed a method of computing special values of a class zeta function of a totally real cubic field. Byeon [1] exploited this result to give

$$
\zeta_{K}(-1, C)=-\frac{p(m)}{2^{3} * 3^{3} * 5 * 7}
$$

where $p(m)=m^{6}+9 m^{5}+55 m^{4}+195 m^{3}+544 m^{2}+876 m+840, K$ is the simplest cubic field corresponding to $m$, and $C$ denotes the principal ideal class. We remark that $\zeta_{K}(-1, C)=\zeta_{K}(-1)$, if $K$ has class number 1 . For $m=-1,1,2,4,7,8,10$ which are all the values of $m$ such that $K$ has class number 1 , our result coincides with Byeon's result.

Remark 5.2. In 7], Siegel gave three examples for the zeta values of totally real number fields. In the last example, Siegel computed that

$$
\zeta_{K}(2)=\frac{2^{3}}{3 * 7^{4}} \pi^{6}
$$

where $K$ is the maximal real subfield of cyclotomic field, $\mathbb{Q}\left(\zeta_{7}\right)$. We note that this field is the same as the simplest cubic field with $m=-1$. By our computation, we have

$$
\zeta_{K}(-1)=-\frac{1}{21}
$$

By the functional equation, our result and Siegel's result coincide. More generally, we have (cf. 4]) that if $D=m^{2}+3 m+9=p$ is a prime, our simplest cubic field corresponding to $m$ is the cubic subfield of $\mathbb{Q}\left(\zeta_{p}\right)$. Therefore our computation contains a table of zeta values of cubic subfield of $\mathbb{Q}\left(\zeta_{p}\right)$, where $p$ runs over primes of the form $m^{2}+3 m+9$. 
TABLE 1. Values of zeta functions of the first one hundred simplest cubic fields

\begin{tabular}{|c|c|c|c|c|}
\hline $\mathrm{m}$ & $\mathrm{D}$ & $-21 \zeta_{K}(-1)$ & $8190 \zeta_{K}(-3)$ & $-3591 \zeta_{K}(-5)$ \\
\hline-1 & 7 & 1 & $3^{2} * 337$ & $3 * 19 * 7393$ \\
\hline 1 & 13 & 7 & $2^{2} * 11 * 43 * 113$ & $2^{2} * 11 * 8459933$ \\
\hline 2 & 19 & $3^{*} 7$ & $13 * 37 * 53 * 127$ & $733 * 33830759$ \\
\hline 4 & 37 & $3 * 7^{2}$ & $3^{4} * 29 * 43 * 3433$ & $3^{2} * 769 * 5478511391$ \\
\hline 7 & 79 & $7^{*} 199$ & $3^{2} * 5 * 12491 * 124799$ & $3^{2} * 19 * 1091 * 6661 * 9349 * 13721$ \\
\hline 8 & 97 & $7^{*} 367$ & $5 * 23 * 1783 * 1439381$ & $11 * 138582878707283203$ \\
\hline 10 & 139 & $5^{2} * 7 * 43$ & $3^{3} * 13 * 3659 * 2851327$ & $3 * 13 * 214201811 * 9547468279$ \\
\hline 11 & 163 & $2^{2} * 7 * 491$ & $2^{2} * 7 * 19 * 89 * 193 * 1226857$ & $2^{2} * 31 * 3709558534651158701$ \\
\hline 13 & $7^{*} 31$ & $3^{*} 89^{*} * 113$ & $5 * 16562502882041$ & $149 * 76308073 * 941711501441$ \\
\hline 14 & $13^{*} 19$ & $3^{*} 7^{*} 19^{*} 109$ & $23 * 263 * 33871899353$ & $7 * 17 * 43 * 73 * 97 * 1227919193250349$ \\
\hline 16 & 313 & $7 * 11 * 1307$ & $43 * 109 * 81553 * 2826371$ & $77158038781 * 7803617145641$ \\
\hline 17 & 349 & $2^{6} * 3 * 643$ & $1747 * 9649 * 136679953$ & $5^{2} * 113 * 8747 * 554117 * 145617822941$ \\
\hline 19 & $7^{*} 61$ & $3 * 5^{2} * 7^{2} * 61$ & $3 * 67 * 47053638267793$ & $3^{2} * 7 * 291060286406388642904463$ \\
\hline 20 & $7^{*} 67$ & $3^{2} * 32999$ & 18239333904161593 & $670333 * 35680459 * 2151776396311$ \\
\hline 22 & $13^{*} 43$ & $71^{*} 6977$ & $5 * 11 * 491 * 2307105606107$ & $5 * 2221 * 2719 * 61493 * 693223 * 275750779$ \\
\hline 23 & 607 & $2^{2} * 13 * 53 * 239$ & $2^{2} * 5 * 7 * 11 * 23 * 119923 * 26135957$ & $2^{2} * 16087 * 13650960177131550855541$ \\
\hline 25 & 709 & $2^{4} * 63313$ & $7 * 601 * 78198106960261$ & $7 * 23 * 37 * 59^{2} * 1187 * 197033309231552533$ \\
\hline 26 & $7^{*} 109$ & $2^{3} * 3 * 5 * 11987$ & $199 * 283 * 9807732170543$ & $29 * 277 * 1259 * 1075352008183959644609$ \\
\hline 28 & 877 & 1954357 & $7 * 17 * 2378473 * 5150532091$ & $1163 * 1184303536883 * 36523018721741$ \\
\hline 29 & 937 & $2 * 3^{2} * 7 * 11 * 1667$ & $2 * 11^{2} * 1613 * 5933822299921$ & $2 * 13 * 43 * 16703 * 554573 * 10058973405480353$ \\
\hline 31 & 1063 & $3^{2} * 61 * 6947$ & $2^{2} * 3 * 13 * 127 * 283880002753163$ & $2^{2} * 3 * 104092933933 * 334151629777440823$ \\
\hline 32 & 1129 & 4092029 & $719 * 11878098993357673$ & $7 * 59 * 68045729 * 101323217 * 284306417491$ \\
\hline 34 & $7^{*} 181$ & $37^{*} 43 * 3719$ & $2 * 3 * 7 * 456028428993552851$ & $2 * 3^{2} * 73 * 2346733 * 3260563 * 286291222738333$ \\
\hline 35 & $13 * 103$ & $2^{7} * 3 * 41 * 443$ & $2^{2} * 3 * 13 * 89 * 109 * 18630840320461$ & $2^{2} * 3 * 5^{2} * 17622967348181675982056803609$ \\
\hline 37 & 1489 & $5^{2} * 11 * 37369$ & $2 * 3 * 61 * 3769193 * 43027272209$ & $2 * 3 * 43 * 13054889 * 5047454508002855360303$ \\
\hline 38 & 1567 & $3^{*} 3663089$ & $1311847 * 3364513 * 19201327$ & $5 * 13^{2} * 317 * 35999 * 3091476759796261264391$ \\
\hline 40 & $7^{*} 13^{*} 19$ & $3^{*} 5^{*} 241^{*} 4127$ & $5 * 690629 * 48882455098879$ & $4473971 * 19663857147270005853525581$ \\
\hline 43 & 1987 & $3^{2} * 11 * 257 * 863$ & $2 * 8311 * 98028449 * 274128131$ & $2 * 11 * 37 * 67 * 73 * 9349 * 70912913 * 153913652563969$ \\
\hline 44 & $31 * 67$ & $2^{3} * 173 * 19373$ & $2^{2} * 3 * 11^{2} * 89 * 337 * 571 * 3449 * 7109671$ & $2^{2} * 3^{2} * 323058826847 * 56864372241656924759$ \\
\hline 46 & $31 * 73$ & $7^{2} * 29 * 25943$ & $9649 * 66721 * 213263 * 8118853$ & $87810367 * 19784024041 * 978025626268781$ \\
\hline 47 & $7^{*} 337$ & $3^{2} * 5^{2} * 7 * 37 * 643$ & $3 * 7 * 13 * 3121 * 1743502925737697$ & $3 * 311 * 21844013 * 66661255861 * 1974861474997$ \\
\hline 49 & 2557 & $3 * 7 * 439 * 5059$ & $47 * 55541728640468168369$ & $19 * 29 * 582719 * 20278712979050892926590543$ \\
\hline 50 & 2659 & $2 * 27613801$ & $2 * 3 * 563 * 324720829 * 3130337051$ & $2 * 3^{4} * 43 * 67 * 809 * 321631 * 82442490325966470781$ \\
\hline 52 & $19^{*} 151$ & $3^{3} * 5^{3} * 19681$ & $4872547 * 1199410637635117$ & $5 * 7^{2} * 59 * 7282052597 * 219471078986233329581$ \\
\hline 53 & $13^{*} 229$ & $3^{*} 5^{*} 7^{*} 131^{*} 5387$ & $3 * 89 * 2089344583 * 13569011087$ & $3 * 13 * 667673 * 1332146560670126336212698037$ \\
\hline 55 & $7^{*} 457$ & $2 * 41 * 1148177$ & $3^{2} * 1392023258725412966413$ & $3^{2} * 167329 * 2978321 * 4220323 * 4042430674898279$ \\
\hline 56 & 3313 & $3^{2} * 73 * 171929$ & $2^{2} * 59 * 8849 * 7691695039188067$ & $2^{2} * 233 * 130631 * 923960723340243327410960881$ \\
\hline 58 & 3547 & $2 * 3^{3} * 7^{2} * 50359$ & $2 * 3^{2} * 1436274899 * 999085267501$ & $2 * 3 * 13^{2} * 433 * 542712625267643149137794099129$ \\
\hline 59 & $19^{*} 193$ & $2 * 3^{3} * 59 * 44221$ & $2 * 19 * 523 * 176153 * 9304374089077$ & $2 * 4447 * 15271 * 1059986933 * 2386540073717358073$ \\
\hline
\end{tabular}


TABLE 1. Values of zeta functions of the first one hundred simplest cubic fields (continued)

\begin{tabular}{|c|c|c|c|c|}
\hline $\mathrm{m}$ & $\mathrm{D}$ & $-21 \zeta_{K}(-1)$ & $8190 \zeta_{K}(-3)$ & $-3591 \zeta_{K}(-5)$ \\
\hline 61 & $7^{*} 13^{*} 43$ & $2^{3} * 7 * 1361 * 2539$ & $2 * 11 * 19 * 557 * 130604017 * 1694844391$ & $2 * 11 * 29 * 47 * 968490011 * 24170128718591566624981$ \\
\hline 62 & $7^{*} 577$ & $47^{*} 4077299$ & $3 * 17 * 3083 * 407546982398304031$ & $3 * 11 * 59 * 510814649940548529242657677008589$ \\
\hline 64 & 4297 & $2^{2} * 56468261$ & $2^{2} * 37 * 5923 * 12823 * 1550477 * 5669501$ & $2^{2} * 23 * 47 * 23173 * 71257 * 376039 * 731987945025979501$ \\
\hline 65 & $43 * 103$ & $5^{*} 3803^{*} 13669$ & $29 * 37 * 203921 * 878387 * 636038869$ & $13 * 317 * 2045773003 * 325180992724547059981117$ \\
\hline 67 & $37^{*} 127$ & $2^{2} * 487 * 148859$ & $2^{2} * 11 * 40048790711 * 104857345427$ & $2^{2} * 5 * 13 * 1709 * 4219 * 295319 * 9494163975441146519461$ \\
\hline 68 & $7^{*} 691$ & $2^{4} * 20852521$ & $2^{2} * 8831 * 6409766466827819957$ & $2^{2} * 3433 * 4547 * 50993 * 247734713 * 9162170167019153$ \\
\hline 70 & 5119 & 395061967 & $5 * 13 * 19 * 29 * 293 * 32070120083484583$ & $11 * 19 * 449 * 5399 * 605113 * 43965102650163336247483$ \\
\hline 71 & $19 * 277$ & $2^{*} 3^{*} 29^{*} 1259^{*} 2089$ & $2 * 29 * 467 * 209771 * 736657 * 97987523$ & $2 * 19 * 277 * 7349 * 209497 * 1128697545302716610226713$ \\
\hline 73 & 5557 & 48613047 & $2 * 563 * 530837595019567524541$ & $\frac{2 * 2729 * 17099 * 379910561649181 * 937933283457641}{2}$ \\
\hline 74 & $13^{*} 439$ & $5 * 105141151$ & $2^{5} * 33768545449 * 666517986487$ & $2^{3} * 3623 * 1537947554229723133909140280253393$ \\
\hline 76 & $7^{*} 859$ & $113^{*} 6228709$ & $3 * 199 * 1746507571928846478343$ & $3 * 5 * 7 * 103 * 1933 * 95987 * 2156784490871 * 18298248941341$ \\
\hline 77 & $31 * 199$ & $5 * 133569811$ & $2^{3} * 3 * 23 * 257 * 2539 * 74099 * 187417 * 248309$ & $2^{3} * 3 * 11 * 13^{2} * 23 * 4363 * 8423 * 10631 * 261776876349533965283$ \\
\hline 79 & $13^{*} 499$ & 851224189 & $5 * 1381337 * 255345403 * 1002547561$ & $821 * 2215927245348689 * 100278474963067212523$ \\
\hline 80 & $61^{*} 109$ & $2^{3} * 5 * 11^{3} * 23 * 677$ & $2^{2} * 3^{2} * 131 * 239 * 11441887 * 162717310997$ & $2^{2} * 3 * 5 * 439 * 563 * 10711 * 1506532989667378018879889443$ \\
\hline 82 & $7 * 997$ & $7^{*} 1723^{*} 81023$ & $3 * 761 * 14593 * 88450342216204751$ & $3 * 211287341 * 505440799 * 1272556442751141143053$ \\
\hline 83 & $7^{*} 1021$ & $2 * 7 * 13 * 5976947$ & $2^{2} * 1279 * 20323 * 33485655004376611$ & $2^{2} * 11 * 12037233969541386189917635679611287083$ \\
\hline 85 & 7489 & $2^{8} * 11 * 17 * 29 * 863$ & $79 * 61089110061312639682529$ & $7 * 31 * 2287 * 28097 * 5670167297219 * 11201950091570507$ \\
\hline 86 & $79 * 97$ & $2 * 3 * 7 * 19 * 1844207$ & $2^{3} * 233 * 3055527910211944658537$ & $2^{3} * 941263 * 6595143312067 * 22964195433294933523$ \\
\hline 88 & 8017 & $31^{2} * 1511273$ & $11^{2} * 140527 * 457252338043234607$ & $4508771281021307 * 415609747066992341305171$ \\
\hline 89 & $7^{*} 1171$ & $11 * 146890147$ & $3 * 7 * 337 * 2347523 * 546968036265713$ & $3^{2} * 41 * 10007 * 647874887252786071132152969854657$ \\
\hline 91 & 8563 & $3 * 5 * 11 * 11828293$ & $2^{2} * 7 * 193 * 1231177 * 1391189 * 1337318531$ & $2^{2} * 5 * 193435090239370960677626089659653542847$ \\
\hline 92 & $13 * 673$ & $229 * 8567723$ & $19^{2} * 353 * 443 * 42743 * 49639 * 119690407$ & $29 * 1307 * 129265607569746936676799219000646107$ \\
\hline 94 & 9127 & $2 * 3 * 5^{2} * 14530823$ & $2 * 3^{2} * 3019 * 11411 * 19489 * 210407 * 7580051$ & $2 * 3 * 89 * 4038289 * 3618126365534773923284310078919$ \\
\hline 95 & 9319 & $2^{2} * 3^{2} * 7 * 9090947$ & $2 * 3 * 23 * 4567 * 35374410576344175397$ & $2 * 3^{5} * 20185492307257657676699655862201968689$ \\
\hline 97 & $7^{*} 19^{*} 73$ & $2731^{*} 961033$ & $3 * 5 * 17 * 61 * 67^{2} * 109 * 3904483830648433$ & $3^{2} * 5 * 17 * 97919 * 205592831335243722144072088351789$ \\
\hline 98 & 9907 & $17^{*} 457^{*} 354791$ & $2^{4} * 3 * 17 * 23^{2} * 137 * 578555893979838143$ & $2^{3} * 3 * 17 * 19 * 97 * 20609717 * 1240850496785227961591142803$ \\
\hline 101 & 10513 & $2 * 3 * 19 * 32407373$ & $2^{2} * 3 * 331 * 677 * 3863 * 71119 * 70459211369$ & $2^{2} * 3 * 23^{2} * 607 * 395611 * 35005189 * 692544145041062423953$ \\
\hline 104 & $7^{*} 37^{*} 43$ & $9871^{*} 399727$ & $3 * 1085719 * 23840944791443319923$ & $3 * 43 * 44782939 * 1445560552208881 * 8343123581486893$ \\
\hline 106 & $31^{*} 373$ & $5 * 19^{*} 107^{*} 473353$ & $5 * 71 * 2917 * 421973 * 5724853 * 40509583$ & $89 * 77058029 * 15354927047269501815288111433181$ \\
\hline 107 & 11779 & $5^{*} 7^{*} 281^{*} 490579$ & $17 * 37 * 293 * 1018481089 * 612853210009$ & $5^{3} * 109 * 7883 * 42239 * 28449433686441097449132066461$ \\
\hline 109 & $19 * 643$ & $2^{6} * 3^{2} * 5^{3} * 7 * 17 * 613$ & $2^{3} * 19 * 97 * 191 * 52694742964789700339$ & $2^{8} * 5^{2} * 7^{2} * 421 * 1231141 * 83490123413 * 14210937656088181$ \\
\hline 110 & $7^{*} 1777$ & $5 * 73 * 15036509$ & $2^{2} * 17 * 1303 * 5669 * 15661 * 21404408245153$ & $2^{2} * 27551 * 2133337848636208066351041661322901761$ \\
\hline 112 & 12889 & $7^{*} 383^{*} 829^{*} 2753$ & $2 * 107 * 1008797726127389642012353$ & $2 * 773 * 70685777 * 3180414766618890249818740409039$ \\
\hline 113 & $13^{*} 1009$ & $2^{*} 83^{*} 38685259$ & $2 * 73 * 89 * 487 * 36445741 * 1058171951659$ & $2 * 17 * 245277829556100359 * 50542392001722969955391$ \\
\hline 115 & $37^{*} 367$ & $3^{2} * 793909517$ & $1759 * 71971 * 2456393826949277927$ & $8093 * 20173 * 6848789 * 627157393537 * 879607085547023$ \\
\hline 116 & $19^{*} 727$ & $947^{*} 1721^{*} 5237$ & $397 * 10123805161 * 87557778932609$ & $13 * 31 * 107 * 152940671615593 * 112885239751875897546133$ \\
\hline 121 & 15013 & $2 * 3^{2} * 109 * 5669207$ & $2^{4} * 1483835047 * 26577311740532803$ & $2^{4} * 41 * 2837447687141811961372180943339791141217$ \\
\hline 122 & 15259 & $2^{2} * 3^{2} * 227 * 653 * 1931$ & $3 * 7^{2} * 1611428063939 * 2970462553679$ & $3 * 225402687410705729 * 3290939843348111525889781$ \\
\hline 127 & 16519 & $2^{*} 37^{*} 223^{*} 271^{*} 2903$ & $2^{4} * 3^{2} * 7 * 19^{2} * 191 * 1567 * 11259236068652129$ & $2^{4} * 3 * 110967014115947068405778374120360190687371$ \\
\hline
\end{tabular}


TABLE 1. Values of zeta functions of the first one hundred simplest cubic fields (continued)

\begin{tabular}{|c|c|c|c|c|}
\hline $\mathrm{m}$ & $\mathrm{D}$ & $-21 \zeta_{K}(-1)$ & $8190 \zeta_{K}(-3)$ & $-3591 \zeta_{K}(-5)$ \\
\hline 130 & 17299 & $2 * 691^{*} 10685029$ & $2^{2} * 179 * 1193 * 851229091 * 2329032723197$ & $2^{2} * 129998635880941 * 17015528241242907885387499001$ \\
\hline 133 & 18097 & $2^{*} 17^{*} 61^{*} 269^{*} 30223$ & $7 * 317 * 1747 * 598994082883257615721$ & $22422527167 * 164280574059307 * 3944708488276557173$ \\
\hline 134 & 18367 & 17748484291 & $2 * 31 * 41579 * 999277278409878150031$ & $2 * 11161 * 766127778111278214348391073812463709731$ \\
\hline 136 & 18913 & $2^{2} * 5 * 239 * 4529563$ & 3174678346344873370589816419 & $67 * 863 * 34250691863 * 40991015742997 * 290804843257813$ \\
\hline 140 & 20029 & $3^{*} 7^{*} 13^{*} 17^{*} 4838063$ & $2^{2} * 3^{2} * 71 * 1847806907774287462772183$ & $2^{2} * 3 * 97 * 197293 * 2398243 * 80520645458171421266738272043$ \\
\hline 142 & 20599 & $2^{4} * 3 * 131 * 311 * 13523$ & $5 * 7 * 571 * 1213 * 179909 * 1319533658614187$ & $97 * 431 * 3259 * 33773 * 13122827414382010881591945190163$ \\
\hline 143 & 20887 & $2 * 5 * 2713 * 954847$ & $2^{3} * 11 * 1753 * 8035793 * 5110448131339981$ & $2^{4} * 653 * 22229 * 87317 * 8094756331 * 428542865890674920449$ \\
\hline 155 & 24499 & $2^{2} * 101 * 104228587$ & $5^{2} * 2775107 * 7115791 * 39196716864499$ & $7 * 13 * 67 * 6989293 * 55307177005420027 * 172549789664464673$ \\
\hline 158 & 25447 & $223 * 2017 * 104911$ & $5 * 2757037 * 5407561 * 9290233 * 36450241$ & $5 * 17^{2} * 427315325862435484252362369926770131512869$ \\
\hline 163 & 27067 & $2^{2} * 14626374283$ & $7^{2} * 157 * 42178439 * 138575623 * 865517501$ & $7 * 4569406109 * 38063279570318234990797412471959643$ \\
\hline 164 & 27397 & $3^{*} 69247^{*} 279557$ & $5 * 7 * 197 * 2099 * 35743339 * 81808157288513$ & $239 * 307 * 51202087 * 370273282523633604705350933056517$ \\
\hline 169 & 29077 & $2 * 3 * 43 * 53 * 5160767$ & $3 * 11 * 17 * 73 * 1567641825047747473525643$ & $3^{2} * 13147 * 6669983 * 7226057550873961 * 469422685830334319$ \\
\hline 172 & 30109 & $2 * 3^{4} * 5 * 7^{3} * 277169$ & $2^{2} * 3 * 101747 * 67109061379176186974579$ & $2^{2} * 3 * 293 * 3163 * 8089 * 43674699384570741974762236223409271$ \\
\hline 175 & 31159 & $5^{*} 7^{*} 2424763087$ & $3^{3} * 19 * 47 * 79 * 54682554396150587382661$ & $3^{3} * 17 * 151 * 373 * 86509 * 6900841 * 371167560006254642622403129$ \\
\hline 176 & 31513 & $2 * 3^{3} * 11 * 17 * 443 * 21611$ & $2 * 3 * 1091 * 38867 * 444778684474815104501$ & $2 * 3 * 11 * 67 * 211 * 6952898984106201970657148609528580833047$ \\
\hline 179 & 32587 & $2^{2} * 11 * 43 * 353 * 146801$ & $2 * 7^{2} * 428735646347 * 3392424901609537$ & $2 * 59 * 52027961640131 * 267437762901247 * 5711626833065897$ \\
\hline 182 & 33679 & $229^{*} 1627^{*} 293453$ & $468509 * 383249124771912502820921$ & $71 * 1672381 * 3079124513 * 36859044295362021984809827111$ \\
\hline 197 & 39409 & $2^{5} * 7 * 13 * 149 * 394633$ & $5 * 13 * 1879 * 2017 * 22511 * 80363 * 1209969999751$ & $1787 * 21757 * 52218007 * 653240873 * 57219691957603126607837$ \\
\hline 200 & 40609 & $2^{2} * 7 * 4327 * 1619249$ & $2^{2} * 29 * 43 * 4297 * 207061 * 6895901 * 21742339627$ & $2^{2} * 79 * 118096859687 * 989118903346943 * 2859469032020339041$ \\
\hline 205 & 42649 & $3 * 7 * 10864049089$ & $2^{4} * 2851 * 1445699 * 14230966026770323219$ & $2^{5} * 2680991952303795365657 * 2109475765541940340229423$ \\
\hline 206 & 43063 & 252696953417 & $2 * 373 * 458099774023 * 2946831153113677$ & $2 * 11 * 823 * 33641 * 419897977991 * 787031379684399222464801657$ \\
\hline 212 & 45589 & $2 * 19 * 7447442731$ & $2^{5} * 23 * 1171 * 79537 * 38644787 * 564943119089$ & $2^{3} * 11 * 159853 * 12405168581731 * 17122246624369 * 126100416816631$ \\
\hline
\end{tabular}




\section{REFERENCES}

1. D. Byeon, Special values of zeta functions of the simplest cubic fields and their applications, Proc. Japan Acad. Ser. A. Math. Sci. 74 (1988), 13-15. MR 99c:11141

2. U. Halbritter and M. Pohst, On the computation of the values of zeta functions of totally real cubic fields, J. Number Theory 36 (1990), 266-288. MR 92b:11080

3. H. K. Kim and H. J. Hwang, Values of zeta functions and class number 1 criterion for the simplest cubic fields, Nagoya Math. J. 160 (2000), 161-180. CMP 2001:06

4. J. S. Kim, Determination of class numbers of the simplest cubic fields, to appear in Comm. of the Korean Math. Soc.

5. A. J. Lazarus, The class number and cyclotomy of simplest quartic fields, PhD thesis, University of California, Berkeley, 1989.

6. D. Shanks, The simplest cubic fields, Math. Comp. 28 (1974), 1137-1152. MR 50:4537

7. C. L. Siegel, Berechnung von Zetafuncktionen an ganzzahligen Stellen, Nachr. Akad. Wiss. Göttingen Math.-Phys. Kl. II (1969), 87-102. MR 40:5570

8. L. C. Washington, Class numbers of the simplest cubic fields, Math. Comp. 48 (1987), 371-384. MR 88a:11107

9. L. C. Washington, Introduction to cyclotomic fields, Graduate Texts in Mathematics, 83, Springer-Verlag, New York 1982. MR 85g:11001

10. D. B. Zagier, "On the values at negative integers of the zeta function of a real quadratic field", Enseignement Math. 22 (1976), 55-95. MR 53:10742

Department of Mathematics, Pohang University of Science and Technology, Pohang 790-784, Korea; and School of Mathematics, Korea Institute for Advanced Study, Seoul 130-012, KOREA

E-mail address: hkkim@postech.ac.kr

Department of Mathematics, Pohang University of Science and Technology, Pohang 790-784, KOREA

E-mail address: integer@euclid.postech.ac.kr 